\title{
TU/e Emphoven

\section{Design and performance of a THz emission and detection setup based on a semi-insulating GaAs emitter}

\section{Citation for published version (APA):}

Zhao, G., Schouten, R. N., Valk, van der, N., Wenckebach, W. T., \& Planken, P. C. M. (2002). Design and performance of a THz emission and detection setup based on a semi-insulating GaAs emitter. Review of Scientific Instruments, 73(4), 1715-1719. https://doi.org/10.1063/1.1459095

DOI:

10.1063/1.1459095

Document status and date:

Published: 01/01/2002

\section{Document Version:}

Publisher's PDF, also known as Version of Record (includes final page, issue and volume numbers)

\section{Please check the document version of this publication:}

- A submitted manuscript is the version of the article upon submission and before peer-review. There can be important differences between the submitted version and the official published version of record. People interested in the research are advised to contact the author for the final version of the publication, or visit the $\mathrm{DOI}$ to the publisher's website.

- The final author version and the galley proof are versions of the publication after peer review.

- The final published version features the final layout of the paper including the volume, issue and page numbers.

Link to publication

\section{General rights}

Copyright and moral rights for the publications made accessible in the public portal are retained by the authors and/or other copyright owners and it is a condition of accessing publications that users recognise and abide by the legal requirements associated with these rights.

- Users may download and print one copy of any publication from the public portal for the purpose of private study or research.

- You may not further distribute the material or use it for any profit-making activity or commercial gain

- You may freely distribute the URL identifying the publication in the public portal.

If the publication is distributed under the terms of Article 25fa of the Dutch Copyright Act, indicated by the "Taverne" license above, please follow below link for the End User Agreement:

www.tue.nl/taverne

Take down policy

If you believe that this document breaches copyright please contact us at:

openaccess@tue.nl

providing details and we will investigate your claim. 


\title{
Design and performance of a THz emission and detection setup based on a semi-insulating GaAs emitter
}

\author{
G. Zhao, R. N. Schouten, N. van der Valk, W. Th. Wenckebach, \\ and P. C. M. Planken ${ }^{\mathrm{a}}$ \\ Delft University of Technology, Faculty of Applied Sciences, Department of Applied Physics, Lorentzweg 1, \\ 2628 CJ Delft, The Netherlands
}

(Received 10 December 2001; accepted for publication 21 January 2002)

\begin{abstract}
We have built a relatively simple, highly efficient, $\mathrm{THz}$ emission and detection system centered around a 15 fs Ti:sapphire laser. In the system, $200 \mathrm{~mW}$ of laser power is focused to a $120 \mu \mathrm{m}$ diam spot between two silverpaint electrodes on the surface of a semi-insulating GaAs crystal, kept at a temperature near $300 \mathrm{~K}$, biased with a $50 \mathrm{kHz}$, $\pm 400 \mathrm{~V}$ square wave. Using rapid delay scanning and lock-in detection at $50 \mathrm{kHz}$, we obtain probe laser quantum-noise limited signals using a standard electro-optic detection scheme with a 1-mm-thick (110) oriented ZnTe crystal or a (110) oriented 0.1-mm-thick GaP crystal. The maximum THz-induced differential signal that we observe is $\Delta I / I=7 \times 10^{-3}$, corresponding to a $\mathrm{THz}$ peak amplitude of $95 \mathrm{~V} / \mathrm{cm}$. The $\mathrm{THz}$ average power was measured to be about $40 \mu \mathrm{W}$, to our knowledge, the highest power reported so far generated with Ti:sapphire oscillators as a pump source. The system uses off-the-shelf electronics and requires no microfabrication techniques. (C) 2002 American Institute of Physics.
\end{abstract}

[DOI: 10.1063/1.1459095]

\section{INTRODUCTION}

Terahertz time-domain spectroscopy (THz-TDS) has gained widespread popularity in recent years due to the wide range of applications, both in science and technology. ${ }^{1-4}$ Generally, there are two methods to generate THz pulses. In the first case, called optical rectification, frequency components in the bandwidth of an ultrashort laser pulse are mixed in a nonlinear crystal such as $\mathrm{ZnTe}$ or $\mathrm{GaP}$, to generate a few-cycle THz pulse. ${ }^{5-7}$ In the second, an ultrashort laser pulse illuminates a semiconductor such as GaAs, biased with an electric or a magnetic field, giving rise to an ultrafast current transient which emits a THz pulse. ${ }^{8-10}$ Of the two methods, optical rectification has the inherent advantage of simplicity compared to the emitters based on electrically biased, microfabricated structures. The disadvantage is that the peak electric fields are generally much smaller than the peak electric fields generated by the biased emitters. An alternative to microfabricated antennas, which typically have dimensions of several microns, to several tens of microns, is the large aperture emitter. These typically consist of two electrodes on a semiconductor, separated by a distance larger than the wavelength of the emitted $\mathrm{THz}$ light. In most cases, these emitters are biased with a dc voltage with a maximum determined by the heat which is generated by the electrical current. Large-aperture emitters are used with amplified, pulsed, lasers which cannot be focused to small spot sizes without damaging the material. ${ }^{11-14}$ They are rarely used in combination with low-peak power Ti:sapphire oscillators. ${ }^{9}$

$\mathrm{THz}$ detection systems can be separated into three categories: The first uses bolometers, which are essentially heat detectors. ${ }^{15}$ The second uses electro-optic detection in a crys-

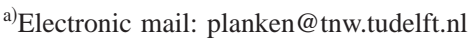

tal such as (110) oriented $\mathrm{ZnTe}$ in which the $\mathrm{THz}$ electric field induces a polarization change on a synchronized probe pulse. ${ }^{16-18}$ The third uses microfabricated antennas on semiconductors such as low-temperature grown GaAs (LT-GaAs) or radiation-damaged $\mathrm{Si}$ on Sapphire which are time gated by a synchronized probe pulse. ${ }^{8,19}$ Of these three detection methods, bolometers have the disadvantage that, for proper operation, they need to be cooled by liquid helium. Electrooptic detection has the great advantage that it measures the amplitude and phase of the $\mathrm{THz}$ electric field, and is inherently simple, but multiple reflections in the detection crystal, and reshaping of the THz pulse due to phase matching issues and absorption, distort the THz pulse..$^{20,21}$ Time-gated microfabricated antennas, finally, also measure the amplitude and phase of a $\mathrm{THz}$ pulse, but require specific expertise to make them using cleanroom facilities. A detailed noise analysis of a THz-TDS systems based on microfabricated antennas, is given in Ref. 8. A similarly detailed analysis for an electrooptic detection scheme is lacking although a comparison between electro-optic detection and detection using microfabricated antennas is given in Ref. 19.

Here, we show that the combination of electro-optic detection and simple, semilarge aperture, biased, semiinsulating GaAs (SI-GaAs) emitters, provides an excellent $\mathrm{THz}$ generation/detection system, which can be used for $\mathrm{THz}$ spectroscopy and imaging up to $6 \mathrm{THz}$. Surprisingly, we find that biased SI-GaAs with simple, silverpaint electrodes can be a very powerful $\mathrm{THz}$ emitter, surpassing the best high power emitters mentioned in literature for systems using Ti: sapphire oscillators. Using a room-temperature pyroelectric detector for absolute power measurements, we find that we generate approximately $40 \mu \mathrm{W}$ of THz power. This number is supported by the value of $95 \mathrm{~V} / \mathrm{cm}$ of the $\mathrm{THz}$ electric field 
a)

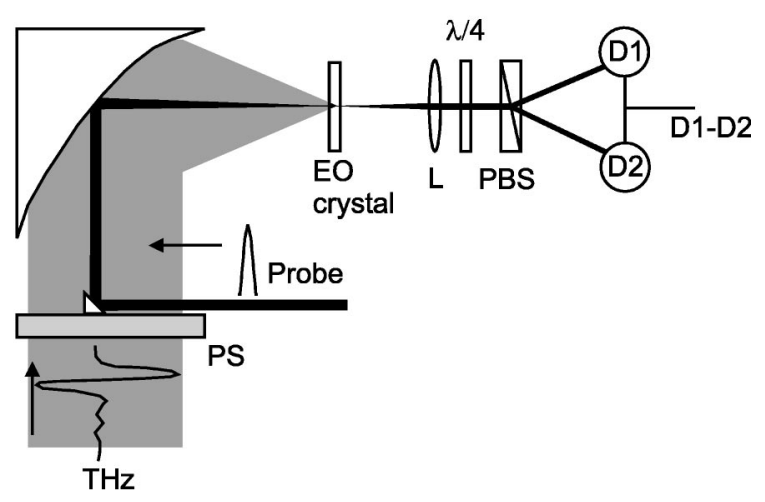

b)

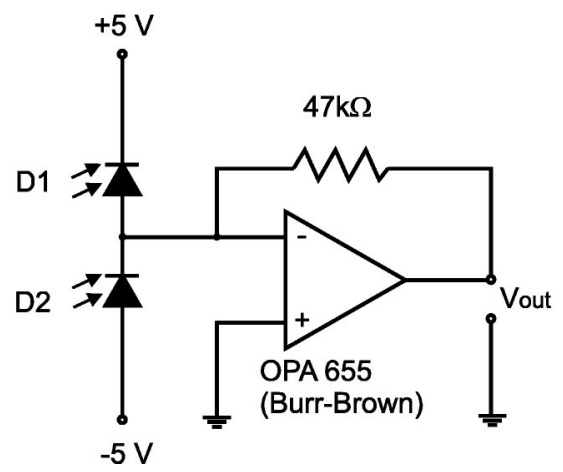

FIG. 1. (a) The THz detection setup, with PS a 2-mm-thick piece of polystyrene foam, $L$ a lens, PBS a polarizing beamsplitter, and $\lambda / 4$, a quarter waveplate. Note the small triangle on the polystyrene plate representing the gold coated prism. (b) Schematics of the differential detector. $D 1$ and $D 2$ are photodiodes of type BPW34 from Siemens.

measured in our electro-optic detection setup. We provide a detailed noise analysis of our setup and demonstrate that our detection limit is determined by probe laser quantum noise only.

\section{EXPERIMENT}

The femtosecond laser used in our experiments is from Femtolasers Produktions, Austria. It generates $450 \mathrm{~mW}$ of average power, and produces 15 fs pulses with a $72 \mathrm{MHz}$ repetition rate and a center wavelength of $775 \mathrm{~nm}$. The emitter is a crystal of SI-GaAs (MCP Wafer Technology Ltd.), which has a dark resistivity of more than $5 \times 10^{7} \Omega \mathrm{cm}$. On the surface of the semiconductor, two crescent-shaped silverpaint electrodes are painted with a smallest separation of 0.4 $\mathrm{mm}$. With this electrode separation, the emitter would qualify as a large aperture emitter, but as the laser beam full width at half maximum (FWHM) spot diameter on the semiconductor is $120 \mu \mathrm{m}$, the term semilarge aperture is more appropriate. $200 \mathrm{~mW}$ of average laser power is used to illuminate the emitter. The sample is glued, using silverpaint, on two 2-mm-thick and 1-cm-wide copper strips, separated by 2 $\mathrm{mm}$. Each copper strip forms a contact to one silverpaint electrode only. The copper strips have two functions: they serve as electrical contacts to the voltage source and they provide a good sink for any heat generated in the GaAs crystal. When the GaAs is illuminated, an average electrical power of about $5 \mathrm{~W}$ is dissipated in the crystal. Preventing the crystal from warming up turned out to be important, because heating of the crystal gives rise to a strong decrease of the emitted $\mathrm{THz}$ amplitude. The copper strips are therefore connected to a water-cooled copper block using an electrically insulating but thermally conductive sheet. A $50 \mathrm{kHz}$, $\pm 400 \mathrm{~V}$ square wave ac bias voltage is applied to the emitter. When the voltage changes from -400 to $+400 \mathrm{~V}$ the $\mathrm{THz}$ signal changes sign as well. Thus, the signal detected by the lock in is twice the signal one would get when the applied voltage switches between 0 and $400 \mathrm{~V}$ only. This method to increase the measured signal without actually increasing the $\mathrm{THz}$ amplitude is very similar to the method of polarization modulation recently reported for a system based on a $\mathrm{ZnTe}$ $\mathrm{THz}$ emitter. ${ }^{22}$ A silicon hyperhemispherical lens is glued on the back of the crystal to focus the, initially strongly divergent, emitted radiation in a more forward direction. The optical path of the vertically polarized $\mathrm{THz}$ beam is shown in Fig. 1. After reflecting from several paraboloidal mirrors, the $\mathrm{THz}$ beam passes through a 2-mm-thick piece of polystyrene foam. The polystyrene foam has a refractive index of 1.017 and an absorption coefficient of less than $1 \mathrm{~cm}^{-1}$ for frequencies below $4 \mathrm{THz}$, and is thus nearly completely transparent in this frequency range, while blocking any remaining nearinfrared "leaking" around the emitter. ${ }^{23}$ A small, gold coated, right-angle prism is glued onto the foam. The prism is used to collinearly combine the $\mathrm{THz}$ pulse with a synchronized, time-delayed, horizontally polarized probe pulse. Both the $\mathrm{THz}$ beam and the probe beam are focused onto the detection crystal, using a $5 \mathrm{~cm}$ focal length, gold-coated paraboloidal mirror. The probe beam then passes through a quarter-wave plate and a Wollaston prism. The Wollaston prism separates the two orthogonal polarization components of the probe beam, which are subsequently focused onto a home-built differential detector. The basic schematic of this detector is shown in Fig. 1(b). When there is no THz electric field present, both polarization components have the same intensity and the output signal of the differential detector is zero. When a THz electric field is present, however, the polarization of the probe pulse in the crystal changes from linear to slightly elliptical. This polarization change is registered as an unbalance in the differential detector, proportional to the $\mathrm{THz}$ electric field. By rapidly changing the optical delay between the probe pulse and the $\mathrm{THz}$ pulse, the complete electric field of the $\mathrm{THz}$ pulse is thus measured. The whole setup is enclosed in a box which is purged with dry nitrogen gas to reduce the effects of water vapor absorption.

\section{RESULTS}

In Fig. 2, we plot (top) the $\mathrm{THz}$ electric field, measured using a 1-mm-thick (110) oriented ZnTe electro-optic crystal (Ingcrys Laser Systems Ltd.). This electric-field scan was obtained in a total time of $20 \mathrm{~ms}$. Magnified by a factor of 200 , we show the results of a measurement when no $\mathrm{THz}$ electric field is present, demonstrating the excellent dynamic range of this measurement. In the bottom figure we plot the $\mathrm{THz}$ electric field measured with a 0.1 -mm-thick (110) oriented GaP EO detection crystal (Molecular Technology 


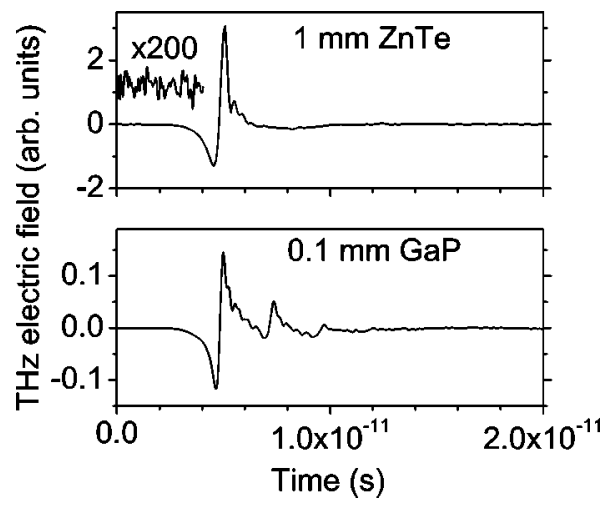

FIG. 2. The top figure shows the measured $\mathrm{THz}$ electric field as a function of time, obtained using a 1-mm-thick (110) oriented ZnTe detection crystal. This measurement corresponds to a total scan time of $20 \mathrm{~ms}$. A signal obtained without a $\mathrm{THz}$ electric field present is magnified 200 times and plotted in the same figure. This signal is displaced vertically for clarity. The bottom figure is a $\mathrm{THz}$ electric field obtained with a 0.1 -mm-thick (110) oriented GaP detection crystal. It is an average of over 2500 scans corresponding to a total scan time of $50 \mathrm{~s}$.

$\mathrm{GmbH})$. The signal is an average over 2500 temporal scans and was obtained in less than a minute. The signal shows several peaks corresponding to multiple $\mathrm{THz}$ reflections in the GaP crystal. A comparison between the two waveforms shows that with the $\mathrm{GaP}$ crystal, we have a larger detection bandwidth at the expense of a 20 times smaller signal compared to the ZnTe crystal. In both $\mathrm{THz}$ waveforms, some weaker oscillations can be seen for longer delays, caused by absorption due to residual water vapor in the nitrogen atmosphere. In Fig. 3 we show the power spectrum of the $\mathrm{THz}$ pulse obtained with $\mathrm{GaP}$, as a function of frequency. From this figure it can be seen that the spectrum of the $\mathrm{THz}$ pulse peaks at roughly $0.5 \mathrm{THz}$. Etalon oscillations with a period of $0.45 \mathrm{THz}$ are visible and are caused by the multiple reflections in the GaP crystal. Frequency components up to 6 $\mathrm{THz}$ are generated. At $1.7 \mathrm{THz}, 2.6 \mathrm{THz}$, and at some other frequencies as well, some absorption lines are visible, associated with the absorption of $\mathrm{THz}$ radiation by residual water vapor.

If $\Delta P=P_{1}-P_{2}$ is the difference in intensity on the two diodes of the differential detector and if $P_{\text {probe }}=P_{1}+P_{2}$ is the intensity of the probe beam, we can express the measured

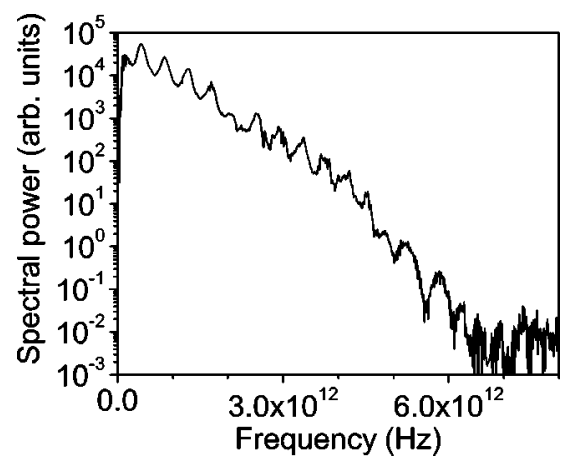

FIG. 3. THz power spectrum, calculated from the THz electric field measurement shown in Fig. 2, taken with the 0.1-mm-thick GaP EO-detection crystal. signal in terms of $\Delta P / P_{\text {probe }}$ to calculate the $\mathrm{THz}$ electric field $E_{T \mathrm{~Hz}}$ from ${ }^{24}$

$$
\frac{\Delta P}{P_{\text {probe }}}=\frac{\omega n^{3} E_{T \mathrm{~Hz}} r_{41} L}{c}
$$

In this equation, $r_{41}=3.9 \mathrm{pm} / \mathrm{V}(\mathrm{ZnTe})$ is the electro-optic coefficient, $n=2.8$ (ZnTe) the near infrared (IR) refractive index, $c$ the speed of light in vacuum, $\omega$ the near-IR angular frequency, and $L$ the length of the crystal.

When $0.1-\mathrm{mm}$-thick $\mathrm{GaP}$ is used as the detection crystal, we measure a peak signal of $\Delta P / P_{\text {probe }}=3.5 \times 10^{-4}$. Using the 1-mm-thick, (110) oriented ZnTe crystal, we measure $\Delta P / P_{\text {probe }}=7 \times 10^{-3}$. This number is higher due to the longer length of the ZnTe crystal and the larger electro-optic coefficient. Based on this, we calculate a peak $\mathrm{THz}$ electric field of $95 \mathrm{~V} / \mathrm{cm}$. With this crystal, we obtain a typical dynamic range of about 3000 for a 35-ps-long $\mathrm{THz}$ electric field scan in a time of $20 \mathrm{~ms}$. An independent measurement of the $\mathrm{THz}$ power is performed using a room-temperature pyroelectric detector, consisting of a detector element (Eltec), without window or coating, in conjunction with a home-built, $900 \mathrm{~Hz}$ bandwidth, amplifier. Because of this relatively low bandwidth, to measure the power, the frequency of the ac bias across the electrodes was lowered to a few hundred $\mathrm{Hz}$. We checked that the $\mathrm{THz}$ amplitude showed no significant change when lowering the bias frequency from $50 \mathrm{kHz}$ to several hundred $\mathrm{Hz}$. The detector was calibrated using the known power of a helium-neon laser. There is some uncertainty as to the response of the detector head at far-infrared wavelengths but it is believed that the response is about $10 \%$ lower in the far infrared. Using this assumption, we deduce an average $\mathrm{THz}$ power of $40 \mu \mathrm{W}$. As mentioned before, about $5 \mathrm{~W}$ of electrical power is dissipated in the sample. To exclude the possibility that the partial conversion of this power into incoherent $\mathrm{THz}$ radiation is responsible for the measured power signal, we also operated the laser in continuous wave (cw) mode. In this mode, the average laser power on the sample is about $250 \mathrm{~mW}$ and the average electrical power dissipated in the sample is also about $5 \mathrm{~W}$, similar to the power dissipated when the laser operates in femtosecond mode. This means that in both modes of operation roughly equal amounts of incoherent thermal $\mathrm{THz}$ radiation, should there be any, are generated. However, in the cw mode of operation, however, no detectable signal was measured with the pyroelectric detector, confirming that the $40 \mu \mathrm{W}$ signal measured with the femtosecond pulses on the emitter indeed corresponds with the coherent $\mathrm{THz}$ pulses only. To our knowledge, this value, supported by the large value of $\Delta P / P_{\text {probe }}$, represents the strongest $\mathrm{THz}$ signal reported in literature using a Ti:sapphire oscillator as a pump source. ${ }^{25-28}$

In Fig. 4, we plot the measured THz peak amplitude as a function of the peak-peak value of the applied bias voltage. The bias-voltage dependence of the THz signal is exponential at first, but shows a trend towards saturation for peakpeak bias values of $300 \mathrm{~V}$ and higher. For similar emitters, increasing the voltage above $400 \mathrm{~V}$ gave rise to sparking 


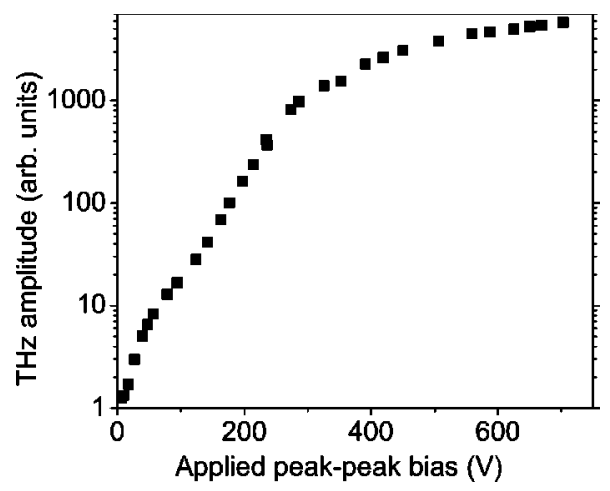

FIG. 4. Measured $\mathrm{THz}$ peak amplitude as a function of the applied bias voltage. A peak-peak voltage of $500 \mathrm{~V}$ corresponds to a voltage swing from -250 to $+250 \mathrm{~V}$ of the square wave.

from one electrode to the other across the surface of the semiconductor, leading to irreversible, visible damage.

The noise properties of the detection setup determine to a large extent whether the signal-to-noise of the setup can be improved or not. The theoretical lower limit of the detector is the shot-noise (SN) limit. With $4.65 \mathrm{~mW}$ of average probe power on each photodiode, having a response of $0.49 \mathrm{~A} / \mathrm{W}$, an average current of $2.28 \mathrm{~mA}$ is generated. The rms shotnoise current fluctuations, expressed in $\mathrm{A} \mathrm{Hz}^{-1 / 2}$, can be calculated according to

$$
\sqrt{\frac{\overline{\left(\Delta I_{\mathrm{SN}}\right)^{2}}}{B}}=\sqrt{2 q I_{0}},
$$

with $q$ the elementary charge, $I_{0}$ the current in the diodes, and $B$ the measurement bandwidth in $\mathrm{Hz}$. We find a value of $\sqrt{\overline{\left(\Delta I_{\mathrm{SN}}\right)^{2}} / B}=2.7 \times 10^{-11} \mathrm{~A} \mathrm{~Hz}^{-1 / 2}$ for each diode. For the two photodiodes combined, we obtain $\sqrt{2 \overline{\left(\Delta I_{\mathrm{SN}}\right)^{2}} / B}=3.82$ $\times 10^{-11} \mathrm{~A} \mathrm{~Hz}^{-1 / 2}$. What is observed in the experiment is the shot-noise voltage $V_{\mathrm{SN}}$ generated across the $47 \mathrm{k} \Omega$ resistor shown in Fig. 1(b). We calculate $V_{\mathrm{SN}}=1.79$ $\times 10^{-6} \mathrm{~V} \mathrm{~Hz}^{-1 / 2}$. We plot this number as the horizontal dashed line in Fig. 5. In the same figure, we also plot the measured noise spectrum of this detector, using a Stanford Research Systems SR760 spectrum analyzer, in the range between 1 and $100 \mathrm{kHz}$. Our THz signals are modulated and detected at $50 \mathrm{kHz}$ and this is, therefore, the range in which we have to compare the noise measurement and calculation.

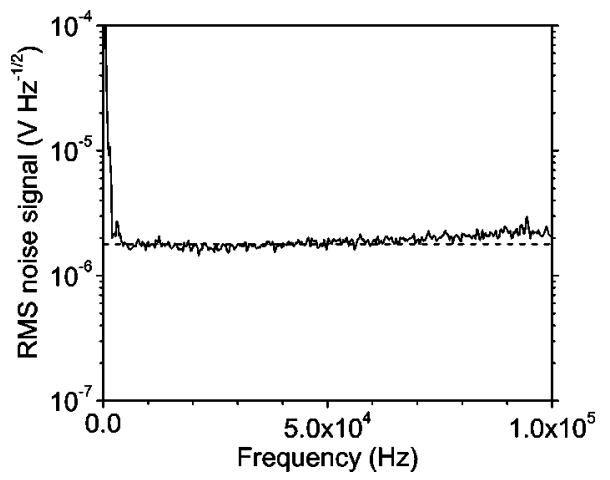

FIG. 5. Measured (solid line) and calculated (dashed line) noise spectra from our differential detector.
As can be seen from the figure, the agreement in this range is excellent, indicating that the detector truly operates in the shot-noise limit.

We define the noise-equivalent differential power (NEDP) as the minimum detectable probe power difference $\Delta P_{\text {NEDP }}$, capable of generating a current equal to the rms shot-noise current. The smallest detectable relative power fluctuations can then also be expressed in terms of

$$
\frac{\Delta P_{\mathrm{NEDP}}}{\left(P_{\text {probe }} \sqrt{B}\right)}=\frac{\sqrt{2 \overline{\left(\Delta I_{\mathrm{SN}}\right)^{2}}}}{\left(2 I_{0} \sqrt{B}\right)} .
$$

In this equation, the term on the right-hand side constitutes the relative current fluctuations in which the shot-noise $\sqrt{\left[2\left(\Delta I_{\mathrm{SN}}\right)^{2}\right]}$ is the combined shot noise of the two photodiodes. Equation (3) is evaluated by using the corresponding currents in the differential detector: An average current of $I_{0}=2.28 \mathrm{~mA}$ runs through each photodiode. This is the current generated by a power $\frac{1}{2} P_{\text {probe }}$. With $\sqrt{2 \overline{\left(\Delta I_{\mathrm{SN}}\right)^{2}} / B}$ $=3.82 \times 10^{-11} \mathrm{~A} \mathrm{~Hz}^{-1 / 2}$, we find $\Delta P_{\mathrm{NEDP}} /\left(P_{\text {probe }} \sqrt{B}\right)=8.4$ $\times 10^{-9} \mathrm{~Hz}^{-1 / 2}$. A typical, $40 \mathrm{ps}$ long scan of a $\mathrm{THz}$ electric field obtained in $20 \mathrm{~ms}$ with a $20 \mathrm{kHz}$ bandwidth, gives $\Delta P_{\mathrm{NEDP}} /\left(P_{\text {probe }}\right)=1.2 \times 10^{-6}$. From Eq. (3), using Eq. (1), we can also calculate the noise-equivalent $\mathrm{THz}$ electric field (NEF) $E_{\mathrm{NEF}}$, the $\mathrm{THz}$ electric field capable of generating a signal equal to the NEDP:

$$
\frac{E_{\mathrm{NEF}}}{\sqrt{B}}=\frac{\Delta P_{\mathrm{NEDP}}}{\left(P_{\text {probe }} \sqrt{B}\right)} \frac{c}{\omega n^{3} r_{41} L} .
$$

From this equation we calculate $E_{\mathrm{NEF}} / \sqrt{B}$ $=130 \mu \mathrm{V} \mathrm{cm}^{-1} \mathrm{~Hz}^{-1 / 2}$.

The coherent probe beam, being a stream of photons, also has shot noise (sometimes referred to as quantum noise). The resulting rms relative fluctuations of the number of photons in the probe beam, as would be measured in a spectrum analyzer, using a detector with a $100 \%$ quantum efficiency and a bandwidth $B$, are given by ${ }^{29}$

$$
\frac{\Delta N_{\nu}}{N_{\nu} \sqrt{B}}=\sqrt{\frac{4 \pi \hbar c}{P_{\text {probe }} \lambda}},
$$

with $\Delta N_{\nu}=\sqrt{\overline{N_{\nu}^{2}}}$ the probe beam photon fluctuations, $N_{\nu}$ the number of photons in the probe beam, and $\lambda$ the wavelength of the probe. We find $\Delta N_{\nu} /\left(N_{\nu} \sqrt{B}\right)=7.3 \times 10^{-9} \mathrm{~Hz}^{-1 / 2}$. Using the real quantum efficiency of our detector of $\eta=80 \%$, the predicted value for the relative current fluctuations is then $\sqrt{2 \overline{\left(\Delta I_{\mathrm{SN}}\right)^{2}}} /\left(2 I_{0} \sqrt{B}\right)=\Delta N_{\nu} /\left(N_{\nu} \sqrt{\eta B}\right)=8.2 \times 10^{-9}$ $\mathrm{Hz}^{-1 / 2}$, in agreement with the number $8.4 \times 10^{-9} \mathrm{~Hz}^{-1 / 2}$, found earlier. This demonstrates that probe laser quantum noise is the dominant noise source.

Finally, for completeness, we can calculate what the lowest detectable $\mathrm{THz}$ power is in our setup. The maximum peak $\mathrm{THz}$ electric field is $95 \mathrm{~V} / \mathrm{cm}$, corresponding to a $\Delta P / P_{\text {probe }}=7 \times 10^{-3}$. Using the value $\Delta P_{\mathrm{NEDP}} /\left(P_{\text {probe }} \sqrt{B}\right)$ $=8.4 \times 10^{-9} \mathrm{~Hz}^{-1 / 2}$ and assuming a reasonable measurement bandwidth of $1 \mathrm{~Hz}$, we obtain $\Delta P_{\mathrm{NEDP}} / P_{\text {probe }}=8.4$ 
$\times 10^{-9}$. We thus have a dynamic range of $8.3 \times 10^{5}$ in electric field, corresponding to a dynamic range of $6.9 \times 10^{11}$ in $\mathrm{THz}$ power. As the THz power associated with the $95 \mathrm{~V} / \mathrm{cm}$ is $40 \mu \mathrm{W}$, the minimum detectable $\mathrm{THz}$ power is 5.8 $\times 10^{-17} \mathrm{~W}$, a number somewhat better than the number reported by van Exter et al. in Ref. 8, using microfabricated antennas as $\mathrm{THz}$ detectors.

There are two reasons why our emitter generates relatively powerful THz pulses. First, we use nearly $200 \mathrm{~mW}$ of optical power between the electrodes. In contrast, on microfabricated antennas, a few tens of $\mathrm{mW}$ is the maximum optical power that one can typically allow on the emitter due to the necessarily smaller laser spot size. Second, compared to large-aperture emitters, we can apply larger electric fields. This is possible because we keep the emitter at a temperature of roughly $300 \mathrm{~K}$, and because we apply the bias as a square wave; this increases the peak amplitude at which breakdown in air occurs. It should be noted, however, that a higher $\mathrm{THz}$ power does not necessarily mean a higher efficiency, defined as the $\mathrm{THz}$ power divided by the laser power. For microfabricated antennas, applied electric fields can be strongly enhanced near sharp electrode tips, giving rise to reasonably strong $\mathrm{THz}$ pulses with less laser power than we use. ${ }^{25}$

It is interesting to note that our detection setup operates in the shot-noise limit. As long as this is the case, it pays to increase the optical power on the photodiodes as this increases the dynamic range with which the $\mathrm{THz}$ signal is detected. This can be understood from the following argument: When the optical power increases, the current in the diodes increases and thus the measured signal amplitude, which is linearly proportional to the current. The shot-noise current, however, which determines the NEF, is proportional to the square root of the current only. An increase in optical power thus increases the dynamic range. The limit is determined by the specific optical power saturation properties of the photodiodes used.

Our setup is ideally suited for many applications such as gas sensing and $\mathrm{THz}$ imaging. From a full measurement of the $\mathrm{THz}$ electric field, with and without sample in the beam, the absorption coefficient and the refractive index of the sample can be calculated independently. Etalon oscillations can be eliminated as a potential problem by optically contacting the thin (110) GaP crystal onto a thick (100) oriented $\mathrm{GaP}$ substrate. In (100) oriented crystals, $\mathrm{THz}$ detection is not possible. At the same time, in a thick crystal, reflections from the interfaces are separated from the main $\mathrm{THz}$ signal by a longer time interval and can thus more easily be eliminated.

This work was performed as part of the EU TERAVISION program (IST-1999-10154).

${ }^{1}$ D. Grischkowsky, S. Keiding, M. van Exter, and C. Fattinger, J. Opt. Soc. Am. B 7, 2006 (1990).

${ }^{2}$ H. Harde, R. A. Cheville, and D. Grischkowsky, J. Phys. Chem. 101, 3646 (1997).

${ }^{3}$ D. M. Mittleman, R. H. Jacobsen, and M. C. Nuss, IEEE J. Sel. Top. Quantum Electron. 2, 679 (1996).

${ }^{4}$ S. Hunsche, M. Koch, I. Brener, and M. C. Nuss, Opt. Commun. 150, 22 (1998)

${ }^{5}$ X.-C. Zhang, Y. Yin, and X. F. Ma, Appl. Phys. Lett. 61, 2764 (1992).

${ }^{6}$ A. Nahata, A. S. Weling, and T. F. Heinz, Appl. Phys. Lett. 69, 2321 (1996).

${ }^{7}$ A. Bonvalet, M. Joffre, J. L. Martin, and A. Migus, Appl. Phys. Lett. 67, 2907 (1995).

${ }^{8}$ M. van Exter and D. R. Grischkowsky, IEEE Trans. Microwave Theory Tech. 38, 1684 (1990).

${ }^{9}$ J. T. Darrow, B. B. Hu, X.-C. Zhang, and D. H. Auston, Opt. Lett. 15, 323 (1990).

${ }^{10}$ C. Weiss, R. Wallenstein, and R. Beigang, Appl. Phys. Lett. 77, 4160 (2000).

${ }^{11}$ P. K. Benicewicz and A. J. Taylor, Opt. Lett. 18, 1332 (1993).

${ }^{12}$ A. J. Taylor, P. K. Benicewicz, and S. M. Young, Opt. Lett. 18, 1340 (1993).

${ }^{13}$ A. S. Weling, B. B. Hu, N. M. Froberg, and D. H. Auston, Appl. Phys. Lett. 64, 137 (1993).

${ }^{14}$ S.-G. Park, A. M. Weiner, M. R. Melloch, C. W. Siders, J. L. W. Seiders, and A. J. Taylor, IEEE J. Quantum Electron. 35, 1257 (1999).

${ }^{15}$ B. I. Greene, J. F. Federici, D. R. Dykaar, R. R. Jones, and P. H. Bucksbaum, Appl. Phys. Lett. 59, 893 (1991).

${ }^{16}$ Q. Wu and X.-C. Zhang, Appl. Phys. Lett. 67, 3423 (1995).

${ }^{17}$ Q. Wu and X.-C. Zhang, Appl. Phys. Lett. 68, 1604 (1996).

${ }^{18}$ P. U. Jepsen, C. Winnewisser, M. Schall, V. Schyja, S. R. Keiding, and H. Helm, Phys. Rev. E 53, R3052 (1996).

${ }^{19}$ Y. Cai, I. Brener, J. Lopata, J. Wynn, L. Pfeiffer, J. B. Stark, Q. Wu, X.-C. Zhang, and J. F. Federici, Appl. Phys. Lett. 73, 444 (1998).

${ }^{20}$ H. J. Bakker, G. C. Cho, H. Kurz, Q. Wu, and X.-C. Zhang, J. Opt. Soc. Am. B 15, 1795 (1998).

${ }^{21}$ G. Gallot and D. Grischkowsky, J. Opt. Soc. Am. B 16, 1204 (1999).

${ }^{22}$ Q. Chen and X.-C. Zhang, Appl. Phys. Lett. 74, 3435 (1999).

${ }^{23}$ G. Zhao, M. ter Mors, W. T. Wenckebach, and P. C. M. Planken, J. Opt. Soc. Am. B (to be submitted)

${ }^{24}$ P. C. M. Planken, H.-K. Nienhuys, H. J. Bakker, and W. T. Wenckebach, J. Opt. Soc. Am. B 18, 313 (2001).

${ }^{25}$ Y. Cai, I. Brener, J. Lopata, J. Wynn, L. Pfeiffer, and J. Federici, Appl. Phys. Lett. 71, 2076 (1997).

${ }^{26}$ Q. Wu and X.-C. Zhang, Appl. Phys. Lett. 70, 1784 (1997).

${ }^{27}$ R. McLaughlin, A. Corchia, M. B. Johnston, Q. Chen, C. M. Ciesla, D. D. Arnone, G. A. C. Jones, E. H. Linfield, A. G. Davies, and M. Pepper, Appl. Phys. Lett. 76, 2028 (2000).

${ }^{28}$ J. N. Heyman, P. Neocleous, D. Hebert, P. A. Crowell, T. Mller, and K. Unterrainer, Phys. Rev. B 64, 085202 (2001).

${ }^{29} \mathrm{H}$. A. Haus, Electromagnetic Noise and Quantum Optical Measurements (Springer, Berlin, 2000). 